University of Nebraska - Lincoln

DigitalCommons@University of Nebraska - Lincoln

April 2007

\title{
Novel metabolism in Chlamydomonas through the lens of genomics
}

Arthur R. Grossman

Carnegie Institution

Martin Croft

University of Cambridge

Vadim N. Gladyshev

University of Nebraska-Lincoln, vgladyshev@rics.bwh.harvard.edu

Sebeeha S. Merchant

University of California, Los Angeles

Matthew C. Posewitz

Colorado School of Mines

See next page for additional authors

Follow this and additional works at: https://digitalcommons.unl.edu/biochemgladyshev

Part of the Biochemistry, Biophysics, and Structural Biology Commons

Grossman, Arthur R.; Croft, Martin; Gladyshev, Vadim N.; Merchant, Sebeeha S.; Posewitz, Matthew C.; Prochnik, Simon; and Spalding, Martin H., "Novel metabolism in Chlamydomonas through the lens of genomics" (2007). Vadim Gladyshev Publications. 43.

https://digitalcommons.unl.edu/biochemgladyshev/43

This Article is brought to you for free and open access by the Biochemistry, Department of at DigitalCommons@University of Nebraska - Lincoln. It has been accepted for inclusion in Vadim Gladyshev Publications by an authorized administrator of DigitalCommons@University of Nebraska - Lincoln. 


\section{Authors}

Arthur R. Grossman, Martin Croft, Vadim N. Gladyshev, Sebeeha S. Merchant, Matthew C. Posewitz, Simon Prochnik, and Martin H. Spalding 
Published in Current Opinion in Plant Biology 10:2 (April 2007), pp. 190-198. In an issue on the theme of "Genome Studies and Molecular Genetics," edited by Stefan Jansson and Edward S Buckler. doi:10.1016/j.pbi.2007.01.012 Copyright (C) 2006 Elsevier Ltd. Used by permission.

\title{
Novel metabolism in Chlamydomonas through the lens of genomics
}

\author{
Arthur R. Grossman ${ }^{1}$, Martin Croft ${ }^{2}$, Vadim N. Gladyshev³, Sabeeha S. Merchant ${ }^{4}$, Matthew \\ C. Posewitz ${ }^{5}$, Simon Prochnik ${ }^{6}$ and Martin H. Spalding ${ }^{7}$ \\ ${ }^{1}$ Department of Plant Biology, The Carnegie Institution, 260 Panama Street, Stanford, California 94305, USA \\ ${ }^{2}$ Department of Plant Sciences, University of Cambridge, Downing Street, Cambridge, UK \\ ${ }^{3}$ Department of Biochemistry, N151 Beadle Center, University of Nebraska, Lincoln, Nebraska 68588-0664, USA \\ ${ }^{4}$ Department of Chemistry \& Biochemistry, UCLA, Box 951569, Los Angeles, California 90095-1569, USA \\ ${ }^{5}$ Environmental Science \& Engineering Division, Colorado School of Mines, Golden, Colorado 80401, USA \\ ${ }^{6}$ DOE Joint Genome Institute, 2800 Mitchell Drive, Walnut Creek, California 94598, USA \\ 7Department of Genetics, Development and Cell Biology, 1210 Molecular Biology, Iowa State University, Ames, \\ Iowa 50011-3260, USA \\ Corresponding author - Arthur R Grossman, email: arthurg@stanford.edu
}

\begin{abstract}
Chlamydomonas has traditionally been exploited as an organism that is associated with sophisticated physiological, genetic and molecular analyses, all of which have been used to elucidate several biological processes, especially photosynthesis and flagella function and assembly. Recently, the genomics of Chlamydomonas has been combined with other technologies to unveil new aspects of metabolism, including inorganic carbon utilization, anaerobic fermentation, the suite and functions of selenoproteins, and the regulation of vitamin biosynthesis. These initial findings represent the first glimpse through a genomic window onto the highly complex metabolisms that characterize a unicellular, photosynthetic eukaryote that has maintained both plant-like and animal-like characteristics over evolutionary time.
\end{abstract}

\section{Introduction}

Chlamydomonas reinhardtii is a member of the green algal lineage that diverged from the streptophytes approximately one billion years ago. It has served as an outstanding model organism, especially for analyzing eukaryotic chloroplast biology and the biogenesis and action of flagella and basal bodies $[1,2,3]$. Genetic analyses with this organism began in the mid 20th century and developed into sophisticated molecular and genomic technologies for dissecting biological processes. Unique attributes that make Chlamydomonas ideal for dissecting photosynthesis are its ability to grow heterotrophically in the dark by metabolizing exogenous acetate, and its maintenance of a normal green chloroplast that retains the capacity to perform oxygenic photosynthesis when illuminated following growth in the dark. These characteristics have allowed the isolation of a range of mutants in which the function and biogenesis of the photosynthetic apparatus is adversely affected $[1,4]$. Most other photosynthetic organisms and all vascular plants either do not survive or exhibit growth retardation and pigment loss in the absence of photosynthesis. Recent work on photosynthesis in Chlamydomonas has focused on the discovery of molecules that catalyze the assembly of the photosynthetic apparatus and determine the abundance and rate of synthesis of individual complexes, and regulatory molecules that control the distribution of excitation energy (state transitions) or dissipation of excess absorbed light energy (non-photochemical quenching) $[2,5,6]$.

Many molecular technologies have also been applied to studies of Chlamydomonas. The chloroplast and nuclear genomes of this alga are readily transformed [7]. Plasmid, cosmid, and bacterial artificial chromosome (BAC) libraries are available. Methods have been developed to generate and identify tagged mutant alleles. Alleles that are not tagged can be identified by map-based cloning $[8,9]$. Gene function can be evaluated by suppression of specific gene activities using antisense or RNA interference (RNAi) constructs [10], and reporter genes have been developed to identify regulatory factors and sequences that are involved in regulating gene expression [11].

In this review, we discuss how the genomics of Chlamydomonas are being combined with these other technologies to unveil new aspects of metabolism, including inorganic carbon utilization, anaerobic fermentation, the suite and functions of selenoproteins, and the regulation of vitamin biosynthesis. The facts and concepts discussed below represent initial insights into the highly complex metabolisms that characterize a unicellular, photosynthetic eukaryote. 


\section{Current status of the genome and genome resources}

The value of the technologies described above is augmented by the nearly 300,000 expressed sequence tags (ESTs) $[12,13,14]$ and a draft Chlamydomonas genome sequence (http://genome.jgi-psf.org/Chlre3/Chlre3.home.html). Several different Chlamydomonas cDNA libraries were constructed using RNA from cells grown under different environmental conditions (see Table 1; [12]). The EST sequences were assembled on the basis of sequence similarity, paired-end sequence information, and genomic information to generate a set of Unigenes designated ACEGs (for 'Assembly of Contiguous ESTs verified by Genome sequences') (M Jain et al., unpublished). Unigene sets were used to develop both cDNA- and oligonucleotide (70mer)based microarrays $[13,15]$.

The current draft release (version 3.0) of the $C$. reinhardtii genome (a detailed description to be published elsewhere), generated at the Joint Genome Institute (JGI), used genomic DNA from strain CC-503 $\mathrm{cw} 92 \mathrm{mt}+$. A whole-genome shotgun sequencing strategy was used to generate a draft genome sequence assembly (http://genome.jgi-psf.org/Chlre3/Chlre3. info.html). The assembly was constructed from $\sim 3$ million reads (13-fold redundant coverage) that yielded scaffolds containing $121 \mathrm{Mb}$ and gaps of $\sim 15.0 \mathrm{Mb}$ within scaffolds. The assembled sequence was used for $a b$ initio gene prediction, which was assisted by transcriptome data. Over 15000 gene models were predicted and $\sim 4000$ have been manually curated. Genome-associated resources for Chlamydomonas are available at several websites; some presently active sites are given in Box 1.

\section{Novel metabolisms}

Although many aspects of basic metabolism have been explored using Chlamydomonas, the genome has also revealed or augmented our understanding of novel metabolic processes ranging from the acquisition of inorganic carbon to the exploitation of selenocysteine-containing enzymes.

\section{The carbon concentrating mechanism}

The carbon concentrating mechanism (CCM) uses energy to increase intracellular $\mathrm{CO}_{2}$ concentrations, allowing high rates of photosynthetic $\mathrm{CO}_{2}$ fixation when the concentration of external $\mathrm{Ci}$ (where $\mathrm{Ci}$ is $\mathrm{CO}_{2}, \mathrm{HCO}_{3}{ }^{-}$and $\mathrm{CO}_{3}{ }^{-2}$ ) is low. $\mathrm{A} \mathrm{CCM}$ is crucial for Chlamydomonas and most aquatic photosynthetic organisms because of highly variable concentrations of dissolved $\mathrm{Ci}$ in aqueous environments, the impact of $\mathrm{pH}$ on the relative distribution between dissolved $\mathrm{CO}_{2}$ and bicarbonate, and a $10^{4}$ slower rate of diffusion of $\mathrm{CO}_{2}$ in water relative to air. CCM function depends on active $\mathrm{Ci}$ transport and intracellular bicarbonate accumulation, combined with carbonic anhydrase (CA) activity. CA activity facilitates the dehydration of bicarbonate at the site of localization of ribulose1,5-bisphosphate carboxylase/oxygenase (Rubisco), providing near-saturating $\mathrm{CO}_{2}$ concentrations $\left[\mathrm{CO}_{2}\right]$ for carboxylation. Components that are involved in CCM are discussed below and depicted in Figure 1.

The genome of Chlamydomonas has revealed an unexpectedly large number of encoded CAs. Nine putative CAs that are encoded by the Chlamydomonas genome might contribute to $\mathrm{CCM}$, of which only five (CAH1-CAH5) were known previously. CAH1 (synthesized in ambient $\left[\mathrm{CO}_{2}\right]$ ) and $\mathrm{CAH} 2$ (synthesized when $\left[\mathrm{CO}_{2}\right]>1 \%$ ) are in the periplasm. Their roles in

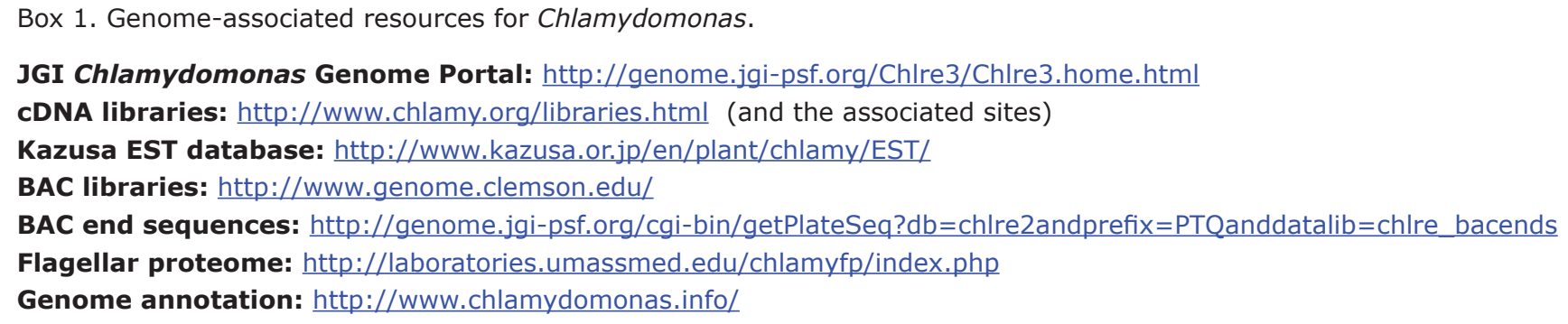

Box 1. Genome-associated resources for Chlamydomonas.

JGI Chlamydomonas Genome Portal: http://genome.jgi-psf.org/Chlre3/Chlre3.home.html

cDNA libraries: http://www.chlamy.org/libraries.html (and the associated sites)

Kazusa EST database: http://www.kazusa.or.jp/en/plant/chlamy/EST/

BAC libraries: http://www.genome.clemson.edu/

BAC end sequences: http://genome.jgi-psf.org/cgi-bin/getPlateSeq?db=chlre2andprefix=PTQanddatalib=chlre bacends

Flagellar proteome: http://laboratories.umassmed.edu/chlamyfp/index.php

Genome annotation: http://www.chlamydomonas.info/

Table 1. Chlamydomonas cDNA libraries constructed using RNA from cells.

$\begin{array}{lll}\text { Library } & \text { Growth conditions } & \text { Project IDs } \\ \text { Core } & \text { High and low }\left[\mathrm{CO}_{2}\right] \text { conditions in minimal salt and acetate-supplemented media } \\ \text { Stress I } & \text { Media devoid of either nitrogen, sulfur or phosphorus } & \mathbf{8 7 4 ,} \mathbf{8 9 4 ,} \mathbf{1 0 2 4} \\ \text { Stress II } & \text { Grown following a shift to different nitrogen sources, under different osmotic conditions, } & \mathbf{9 6 3} \\ & \text { conditions that facilitate } \mathrm{H}_{2} \text { production, and following exposure to cadmium } \\ \text { Stress III } & \text { Copper-free or low-iron medium, high light, anoxic } & \mathbf{1 0 3 1} \\ \text { Deflagellation } & \text { Recovering from deflagellation } & \mathbf{1 1 1 5} \\ \text { Gamete-Zygote } & \text { Developing into gametes and zygotes } \\ \text { S1D2 } & \text { S1D2, a polymorphic strain interfertile with the laboratory strain }\end{array}$




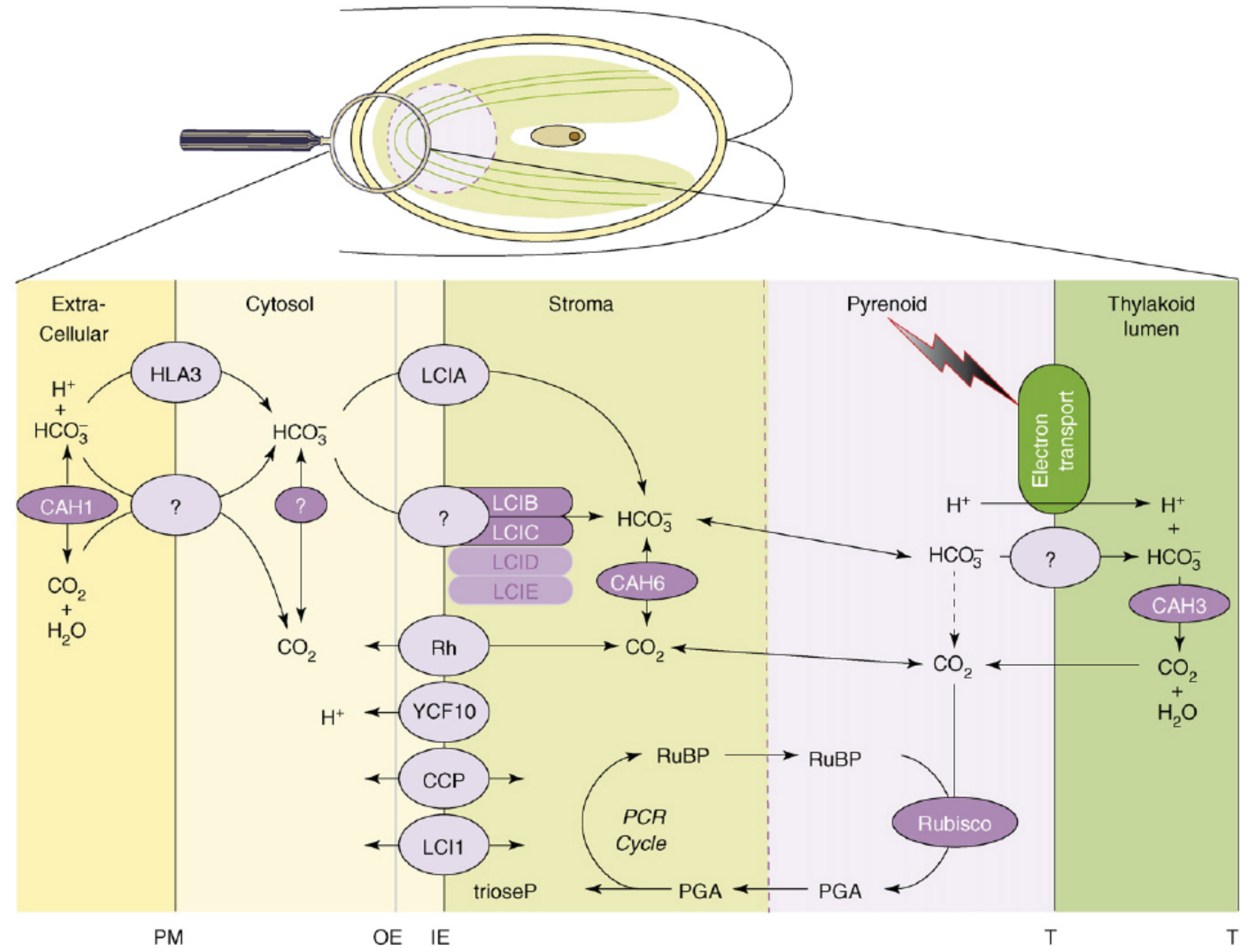

Figure 1. The cell biology of the carbon concentrating mechanism. The movement of inorganic carbon (Ci) from the extracellular space (yellow) to the thylakoid lumen (green) via the cytosol (uncolored) and stroma (light green) is shown. The pyrenoid is a non-membrane-bound compartment (boundary indicated as dashed line) within the stroma, and is the site of Rubisco localization. Membranes that serve as barriers for transport are shown as solid black lines. The lilac and purple ovals indicate transporters and enzymes, respectively, that catalyze steps in $\mathrm{Ci}$ uptake. Question marks indicate unidentified, postulated carriers. Putative $\mathrm{Ci}$ transporters without supporting functional data are indicated without substrates. IE, inner envelope; OE, outer envelope; PM, plasma membrane; T, thylakoid membrane.

the CCM have not been established, but they are probably important when the surrounding milieu is more alkaline $[16,17]$. The $C A H 1$ and $C A H 2$ genes are clustered with other genes that are possibly important for the CCM. CAH3 is located in the thylakoid lumen and its importance was established genetically [18]. The roles of the mitochondrial isoforms CAH4 and $\mathrm{CAH} 5$, which are induced coordinately with $\mathrm{CAH} 1$, are not clear, but these CAs have been implicated in anapleurotic reactions [19]. The newly discovered CA isoforms are CAH6, CAH7, CAH8 and CAH9. The chloroplast stromal location of CAH6 suggests that it might be involved in trapping $\mathrm{Ci}$ as bicarbonate in the alkaline stroma [20], but the cellular locations and functions of the other novel CAs are unknown.
Mutant characterizations have been essential to our understanding of the CCM, revealing both functional and regulatory elements of the system. The cia5 $(\mathrm{ccm} 1)$ mutant of Chlamydomonas cannot acclimate to limiting $\left[\mathrm{CO}_{2}\right]$ and the CIA5 protein is probably a transcription factor that is crucial for acclimation $[21,22]$. The pmpl mutant was reported to be a high-[ $\left[\mathrm{CO}_{2}\right]-$ requiring mutant that has a deficiency in $\mathrm{Ci}$ transport. This strain grew poorly in low or ambient $\left[\mathrm{CO}_{2}\right](\sim 0.04 \%)$ but like the wildtype strain at high $(5 \%)\left[\mathrm{CO}_{2}\right]$ when the $\mathrm{CCM}$ is not necessary. This strain also exhibits normal growth at very low $(\leq 0.01 \%)\left[\mathrm{CO}_{2}\right]$, however, indicating that a multi-tiered regulatory program, controlled by $\mathrm{CO}_{2}$ levels, is crucial for the acclimation of Chlamydomonas to limiting $\left[\mathrm{CO}_{2}\right]$ [23]. Further 
characterizations established three acclimation states: high $\left[\mathrm{CO}_{2}\right]\left(\geq 0.5 \% \mathrm{CO}_{2}\right)$, low $\left[\mathrm{CO}_{2}\right]\left(0.4 \%-0.03 \% \mathrm{CO}_{2}\right)$, and very low $\left[\mathrm{CO}_{2}\right]\left(\leq 0.01 \% \mathrm{CO}_{2}\right)$. The biology of Chlamydomonashas several defining features in each of these three states: in high $\left[\mathrm{CO}_{2}\right]$, genes that are induced by limiting $\mathrm{CO}_{2}$ concentrations are not expressed and the photosynthetic $\mathrm{K}_{1 / 2}\left(\mathrm{CO}_{2}\right)$ is similar to that of the $\mathrm{K}_{\mathrm{m}}\left(\mathrm{CO}_{2}\right)$ of Rubisco. In the low $\left[\mathrm{CO}_{2}\right]$ state, limiting- $\left[\mathrm{CO}_{2}\right]$-regulated genes are induced and photosynthetic $\mathrm{K}_{1 / 2}\left(\mathrm{CO}_{2}\right)$ is markedly decreased. In the very low $\left[\mathrm{CO}_{2}\right]$ state, the photosynthetic $\mathrm{V}_{\text {max }}$ is decreased and the $\mathrm{K}_{1 /}$ ${ }_{2}\left(\mathrm{CO}_{2}\right)$ is further decreased. The abundance of specific transcripts that are associated with $\mathrm{CO}_{2}$ limitation was elevated in both low $\left[\mathrm{CO}_{2}\right]$ and very low $\left[\mathrm{CO}_{2}\right]$ states, suggesting that differences between these two states might arise from a quantitative difference in transcript levels [23], although there might be other, as yet unidentified, genes with expression characteristics that differentiate the two states.

Identification of the defective gene in $p m p 1$ was facilitated by both recognition of its novel 'air-dier' phenotype and the Chlamydomonas draft genome sequence. A tagged mutant gene adl (air dier) was found to be allelic to pmpl. The defect in the adl mutant was shown to be in $L C I B$, a gene activated following exposure of Chlamydomonas to limiting $\left[\mathrm{CO}_{2}\right]$ [24]. This gene is a member of a unique, small gene family consisting of $L C I B, L C I C, L C I D$ and $L C I E$; homologs to these genes were detected only in the genomes of the green microalgae Ostreococcus taurii, Ostreococcus lucimarinus (http://genome.jgi-psf.org/Ost9901_3/Ost9901_3.home.html ; http://genome.jgi-psf.org/Ostta4/Ostta4.home.html) and the colonial alga Volvox carterii. Although presented as being associated with the membrane transport components in Figure 1, LCIB, LCIC and LCID appear to be localized in the plastid, and are soluble polypeptides that function in some unknown way to enable Ci transport.

The draft genome sequence has also allowed the identification of several candidate genes that encode Ci transporters. Some of these genes, $C C P 1, C C P 2$ and $L C I 1$, were previously shown to be responsive to low $\left[\mathrm{CO}_{2}\right]$ and encode putative plastid envelope proteins $[25,26]$, although their role in CCM is still uncertain [27]. Another candidate transporter YCF10 encodes a plastid envelope protein with similarity to CotA in cyanobacteria. Although YCF10 might not be directly involved in $\mathrm{Ci}$ transport, it might influence transport through its proton translocation activity [28]. Additional candidate transporter genes include HLA3/MRP1 and LCIA. LCIA (also called NAR1.2) is induced under limiting- $\left[\mathrm{CO}_{2}\right]$ conditions and encodes a formate/nitrite transporter [29]. LCIA expressed in Xenopus oocytes displays low-affinity bicarbonate transport activity [30] and high-affinity nitrite transport activity, making its role in $\mathrm{Ci}$ uptake uncertain. $H L A 3$, originally identified as a high-light-induced gene and further characterized as being activated during
$\left[\mathrm{CO}_{2}\right]$-limited growth [31], is an $\mathrm{ABC}$ transporter of the multidrug-resistance associated protein (MRP) group.

Another interesting development in recent years is the report that $\mathrm{Rh} 1$ proteins in Chlamydomonas function as $\mathrm{CO}_{2}$ gas channels [32]. Transcripts from the $R H 1$ gene increase in high $\left[\mathrm{CO}_{2}\right]$, suggesting that the encoded proteins might help to provide adequate $\left[\mathrm{CO}_{2}\right]$ for photosynthesis in the absence of a $\mathrm{CCM}$, although the relationship between these proteins and $\mathrm{CO}_{2}$ assimilation in Chlamydomonas has yet to be established. Because of the close relationship between Rh in Chlamydomonas and $\mathrm{Rh}$ blood factors, these findings have generated considerable interest with regard to potential roles of blood $\mathrm{Rh}$ in $\mathrm{CO}_{2}$ transfer [33].

Recently, several genes that are induced by low $\left[\mathrm{CO}_{2}\right]$ were localized to a $75 \mathrm{kbp}$ region [24] of scaffold 15 of version 3.0 of the Chlamydomonas draft genome. This cluster includes the limiting- $\left[\mathrm{CO}_{2}\right]$-induced genes $C C P 2$ and $C C P 1$, which encode plastid envelope proteins of the mitochondrial carrier family of proteins [27], LCID, LCIE, CAH1 and CAH2. The arrangement of the gene models suggests a duplication of an ancestral $L C I-C C P$ gene pair flanked by an ancestral periplasmic $C A H$ gene, but the functional importance of this arrangement, if any, is not yet understood.

\section{Fermentation and hydrogen metabolism}

Anoxic metabolism in Chlamydomonas (Figure 2) utilizes fermentative pathways that are typically present in strict anaerobes to metabolize starch into a variety of fuels, including $\mathrm{H}_{2}$ and ethanol $[34,35,36]$. The use of fermentative pathways that are associated with anaerobic chemotrophs by an oxygenic phototroph was initially considered to be paradoxical; however, it is now apparent that photosynthetic microbes may experience extended periods of anoxia. When Chlamydomonas is grown with limited aeration under low light intensities, $\mathrm{O}_{2}$ consumption by respiration can exceed the rate of photosynthetic $\mathrm{O}_{2}$ production, leading to hypoxia [37], a common condition in the natural environment. Previous biochemical analyses have demonstrated that formate, acetate, and ethanol are major fermentative products formed in parallel with $\mathrm{H}_{2}$ and $\mathrm{CO}_{2}$ ([38, 39]; F Mus et al., unpublished). Trace amounts of lactate and glycerol have also been reported in Chlamydomonas.

The current draft genome for Chlamydomonas has revealed a unique repertoire of proteins that facilitate metabolic acclimation according to energetic fluxes arising from diurnal light/dark cycles [40, 41]. Several of these proteins function in fermentative production of ATP during anaerobiosis. Intriguingly, Chlamydomonas encodes key enzymes that are required for each of the five predominant pathways used to catabolize pyruvate (shown in Figure 2). This metabolic 


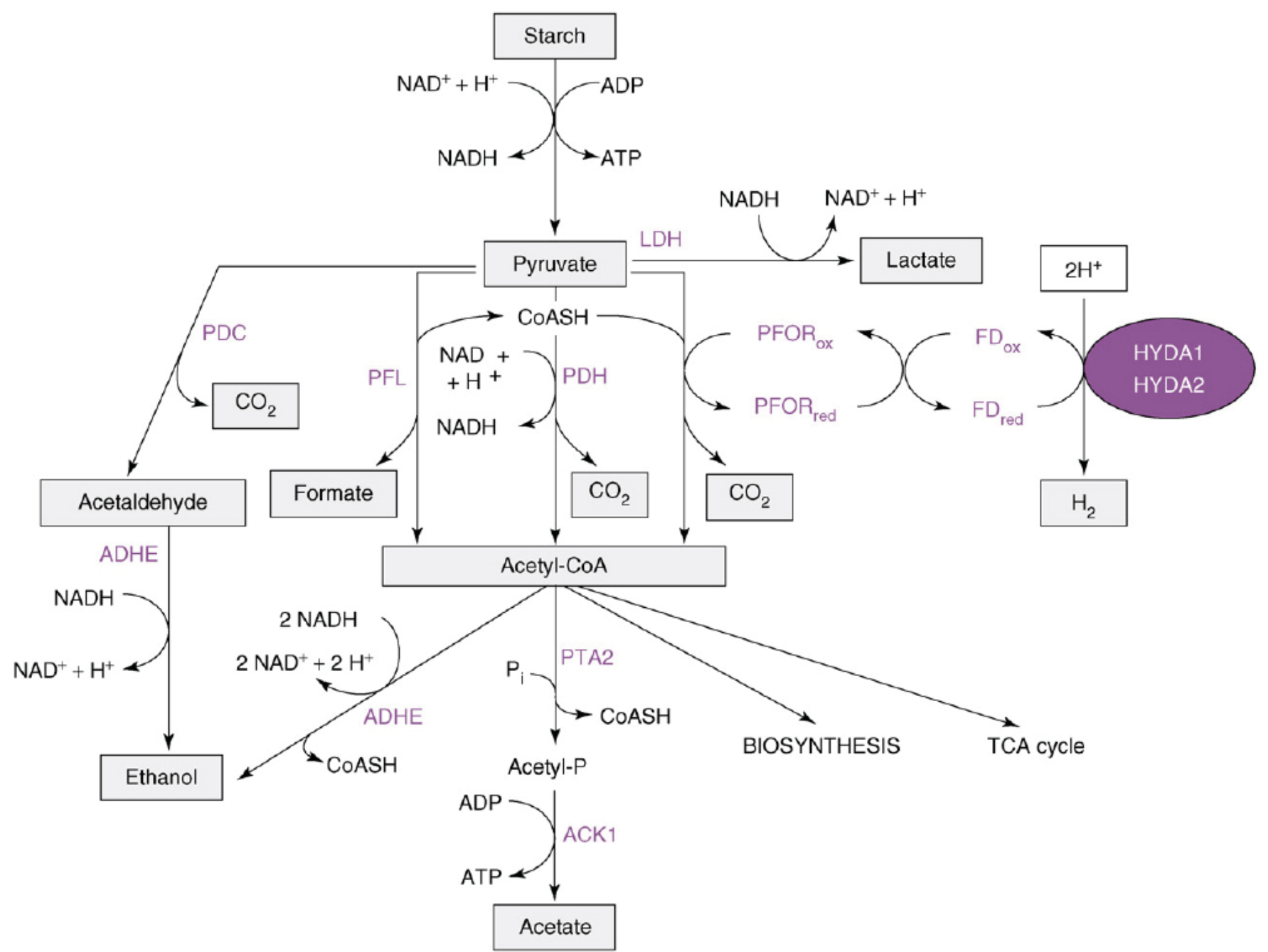

Figure 2. Pyruvate metabolism in Chlamydomonas. The four predominant pathways available in Chlamydomonas for the catabolism of pyruvate are illustrated. Oxygen availability, $\mathrm{NAD}(\mathrm{H})$ and ATP levels influence the partitioning of pyruvate into the appropriate pathways. Parallel processes in mitochondria and the chloroplast are expected for some aspects of pyruvate metabolism. In aerobic conditions, pyruvate is oxidized by the pyruvate dehydrogenase complex (PDH). The NAD ${ }^{+}$that is required for pyruvate production from starch can be regenerated by the respiratory chain (not shown). In anaerobic conditions, pyruvate can be oxidized via pyruvate ferredoxin oxidoreductase (PFOR) yielding acetyl-CoA, reduced ferredoxin and $\mathrm{CO}_{2}$. Alternatively, pyruvate can be cleaved by pyruvate formate lyase (PFL), yielding acetyl-CoA and formate. HYDA1 and HYDA2 are two isoforms of (FeFe)-hydrogenase that oxidize the ferredoxin reduced by the action of PFOR. Pyruvate might also be decarboxylated by the pyruvate decarboxylase (PDC) homolog, resulting in the production of acetaldehyde and $\mathrm{CO}_{2}$. Under hypoxic conditions, $\mathrm{NADH}$ is reoxidized by alcohol/aldehyde dehydrogenase (ADHE) activity. NADH might also be reoxidized by a putative lactate dehydrogenase (LDH). The conversion of acetyl-CoA to acetate and CoA via acetate kinase (ACK1) and phosphotransacetylase (PTA2) results in the fermentative production of ATP.

diversity is extraordinary in a single organism, suggesting marked metabolic flexibility suited for survival in a dynamic environment.

In addition to the pyruvate dehydrogenase complex, which catalyses the oxidation of pyruvate to $\mathrm{CO}_{2}$ and acetyl-CoA (the latter is further oxidized by the TCA cycle under aerobic conditions), the Chlamydomonas genome encodes homologs of pyruvate formate lyase (PFL), pyruvate decarboxylase (PDC) and pyruvate ferredoxin oxidoreductase (PFOR). These en- zymes direct organic carbon into distinct anaerobic pathways that are required to balance levels of NADH oxidation and ATP production during fermentation. PFOR couples the oxidation of pyruvate to acetyl- $\mathrm{CoA}$ and $\mathrm{CO}_{2}$ with the reduction of ferredoxin, which can be used by the [FeFe]-hydrogenase to reduce protons to $\mathrm{H}_{2}$, as shown in Figure 2. The two Chlamydomonas [FeFe]-hydrogenases, encoded by HYDA1 and HYDA2, together with PFOR and the [FeFe]-hydrogenase maturation proteins HYDEF and HYDG [42] are examples of proteins usually only associated with strict anaerobes. 
Interestingly, Chlamydomonas contains both the PFL and PFOR pathways for generating acetyl-CoA, yielding formate and $\mathrm{CO}_{2}$ as co-products, respectively. Conversion of acetylCoA into acetate via the phosphotransacetylase (PTA)/acetate kinase (ACK) pathway results in ATP production. NADH is not reoxidized by the acetate production pathway, however, and consequently the synthesis of ethanol from either acetaldehyde (PDC pathway) or acetyl-CoA (PFL or PFOR pathways) is used to reoxidize NADH to sustain glycolysis. Alcohol and aldehyde dehydrogenase activities are both likely to be catalyzed by the aldehyde/alcohol dehydrogenase (ADHE) homo$\log$ in Chlamydomonas [40, 41]. The presence of both the acetaldehyde and acetyl-CoA pathways for ethanol production and the PFOR and PFL pathways for acetyl-CoA generation is remarkable for a eukaryote. Oxidation of NADH could also be coupled to $\mathrm{H}_{2}$ production, as observed for Clostridia [43].

Interestingly, the Chlamydomonas genome has two copies of $P T A$ and $A C K$. PTA1 and ACK2 are localized to mitochondria, whereas PTA2 and ACK1 are probably in the chloroplast [40]. The cellular location of PFOR is unknown, but it is expected, at least to some extent, to be in the chloroplast with the hydrogenases. PFL, which is encoded by a single Chlamydomonas gene, appears to be in mitochondria and chloroplasts [40], indicating dual-organelle targeting. No homolog of formate dehydrogenase is found on the Chlamydomonas genome, suggesting the absence of formate-hydrogen-lyase activity in Chlamydomonas, in accord with previous biochemical observations [38, 39].

In addition to anaerobiosis in the dark, deprivation of sulfate leads to reduced levels of oxygenic photosynthesis [44], resulting in anaerobiosis in the light [45]. Hence, Chlamydomonas can perform hypoxic fermentative metabolism in the light, creating a unique situation during which oxygenic photosynthesis, respiration and fermentation occur simultaneously. Recent microarray data examining gene expression as Chlamydomonas acclimates to hypoxic conditions has demonstrated that there are marked changes in the expression of genes that are associated with parallel metabolic processes that occur both in mitochondria and chloroplasts (F Mus et al., unpublished). Further examination of these pathways in the different organelles, and of crosstalk among them, will reveal insights that could be leveraged into strategies for engineering metabolism to optimize the production of energy-rich fermentative products. Furthermore, more than $60 \%$ of the differentially expressed genes observed on the microarrays encode putative proteins of unknown function; some are likely to participate in the function and/or regulation of the extraordinary metabolic networks of Chlamydomonas.

\section{Selenoproteins}

Selenoproteins, which contain selenocysteine (Sec), are present in bacteria, Archaea and many Eukarya [46], but not in vascular plants. In bacteria, these proteins are often involved in catabolic processes, whereas in eukaryotes they can have both biosynthetic and antioxidant functions. Chlamydomonas was previously shown to make selenoproteins [47], and a new analysis of the complete genome (VN Gladyshev, unpublished) led to the identification of 12 selenoproteins representing ten families, as well as Sec biosynthesis and insertion machinery, including a Sec-specific elongation factor, selenophosphate synthetase, SECIS-binding protein 2 (SBP2) and a single, previously identified Sec tRNA [48]. It is surprising that so many selenoprotein genes have been found in Chlamydomonas considering that these genes have been lost during the evolution of the streptophytes.

The selenoproteome of Chlamydomonas consists of selenoprotein K (SELK), selenoprotein M (SELM), thioredoxin reductase (TR), methionine-S-sulfoxide reductase (MSRA), glutathione peroxidase 1 (GPX1), glutathione peroxidase 2 (GPX2), selenoprotein U (SELU), selenoprotein T (SELT), selenoprotein W1 (SELW1), selenoprotein W2 (SELW2), selenoprotein $\mathrm{H}$ (SELH) and membrane selenoprotein (MSP). Five of the selenoproteins (TR, MSRA, GPX1, GPX2 and SELM) have homologs that have redox functions. The functions of the remaining seven are not known. Of these seven, five (SELK, SELW1, SELW2, SELH and SELT) have homologs in the human selenoproteome, one (SELU) is found in some animals (fish and birds) and one (MSP) appears to be present only in other unicellular organisms including other green algae (i.e. Volvox carteri and Ostreococcus tauri). Chlamydomonas MSP is predicted to have two Sec and is only the second eukaryotic selenoprotein, after SELP, to utilize more than one Sec. Furthermore, Chlamydomonas has recently been found to contain the most efficient MSRA enzyme known [49]. These data suggest that Chlamydomonas is dependent on the supply of selenium and that this dependence is due to the presence of this trace element in twelve selenoproteins with diverse functions. The selenium demand is probably low, however, because selenoproteins are present at low abundances in Chlamydomonas (and in other organisms). In fact, the growth of Chlamydomonas does not require the specific addition of selenium because enough is included as a contaminant of the other reagents. Given the role of many of the selenoproteins in dealing with oxidative stress, it is possible that the impact of selenium nutrition might be evident only under situations of exacerbated stress, such as excess excitation energy or poor nutrition.

\section{Vitamins}

Eukaryotic algae, like humans, need several vitamins for their growth. Vitamin $\mathrm{B}_{12}$ (cobalamin), vitamin $\mathrm{B}_{1}$ (thiamine) and vitamin $\mathrm{H}$ (biotin) are required by a wide variety of algae, although not all algae require all three vitamins [50]. Chlamydomonas does not, itself, require an external supply of any of these micronutrients, but the recent genome sequence gives us 
some fascinating insights into both vitamin metabolism in algae and the evolution of biosynthetic pathways.

Cobalamin is a tetrapyrrole-derived molecule that is synthesized exclusively by prokaryotic organisms. In humans, the vitamin is required as a cofactor for vitamin $\mathrm{B}_{12}$-dependent methionine synthase. Vascular plants do not require this vitamin because they contain an alternative vitamin $\mathrm{B}_{12}$-independent isoform of methionine synthase. The Chlamydomonas genome sequence has revealed two isoforms of methionine synthase: one is human-like and requires vitamin $\mathrm{B}_{12}$ as a cofactor $(\mathrm{METH})$, whereas the other is plant-like and functions without this vitamin (METE) [51]. This arrangement, in which an individual organism contains two types of methionine synthases, is analogous to the situation in many bacteria, but it has not been observed in vascular plants or animals. The observation that Chlamydomonas has retained both isoforms of the enzyme, and has the ability to differentially control their expression [51], suggests that vitamin $B_{12}$ is only transiently available in the alga's natural environment. The results also suggest specialization and concomitant gene loss in the animal and plant lineages.

Another vitamin, thiamine, plays a key role in intermediary metabolism. The active compound, thiamine pyrophosphate (TPP) is essential for all organisms. Although some algae cannot synthesize TPP de novo, others, such as Chlamydomonas, can [4 and 50]. In all organisms studied to date, TPP is synthesized from a thiazole and a pyrimidine moiety that are joined to form thiamine phosphate, which is then phosphorylated to form TPP. In bacteria, the thiazole moiety is derived from 1deoxy-d-xylulose 5-phosphate, while the pyrimidine moiety is made from 5-aminoimidazole ribonucleotide [52]. Much less is known about thiamine biosynthesis in eukaryotes, but in Saccharomyces cerevisiae it appears that the thiazole and pyrimidine moieties are synthesized through different routes [52]. Interestingly, the genome sequence of Chlamydomonas indicates that the pyrimidine moiety is synthesized by a bacterial route (THIC) and the thiazole moiety made in the same way as in fungi (THI4).

Several of the thiamine biosynthetic genes in Chlamydomonas appear to contain regions that are highly similar to TPP riboswitches $[53,54,55]$. These regulatory elements are structures within the mRNA of individual genes that specifically bind TPP, and in so doing regulate the expression of the cognate gene. Several TPP riboswitches have been studied in bacteria, but little work has been carried out in eukaryotes. Furthermore, EST evidence from the Chlamydomonas genome project suggests that the mRNAs of some thiamine biosynthetic genes are alternatively spliced in riboswitch-containing regions, suggesting that TPP binding to the pre-mRNA might influence splicing. One region of particular interest is in the THIC gene, where EST evidence suggests that the $7^{\text {th }}$ exon can be alternatively spliced. If this exon is included in the mature mRNA, a premature stop codon is incorporated, and a truncated (presumably non-functional) THIC protein is synthesized. If this exon is removed from the mature message, however, a full-length THIC protein is translated. It is therefore intriguing to speculate that TPP can bind to the premRNA and thereby influence the way in which it is spliced.

\section{Conclusions}

The presentation above describes the union of physiological, biochemical and genetic data with near full genome information to reveal new metabolic routes and their control, and to expand our view of known metabolic networks. The examples provided represent only first explorations into topical areas of energy conversion and nutrient utilization. As we learn more about the nutrient requirements of Chlamydomonas (e.g. [56]) and apply developing technologies to examine both metabolite profiles (e.g. [57]) and how nutrient levels alter genomewide expression patterns [13], we will not only learn more about individual pathways but also gain insights into the ways in which these pathway are integrated to optimize energy production and utilization.

\section{Acknowledgements}

We would like to acknowledge that funds supporting part of this work were from National Science Foundation (NSF) grants in support of the Chlamydomonas Genome Project (MCB 0235878 awarded to ARG). The work was also made possible by the efforts of Dan Rokhsar and others at the Joint Genome Institute in generating a draft sequence of the Chlamydomonas genome. All of the authors that have contributed to this article acknowledge support from US Department of Agriculture (USDA), National Institutes of Health (NIH), Department of Energy (DOE), Air Force Office of Scientific Research (AFOSR) and NSF.

\section{References and recommended reading}

Papers of particular interest, published within the annual period of review, have been highlighted as:

- of special interest;

-• of outstanding interest

1 E.H. Harris, Chlamydomonas as a model organism, Annu Rev Plant Physiol Plant Mol Biol 52 (2001), pp. 363-406.

2 J.D. Rochaix, Chlamydomonas, a model system for studying the assembly and dynamics of photosynthetic complexes, FEBS Lett 529 (2002), pp. 34-38.

3 W.J. Snell, J. Pan and Q. Wang, Cilia and flagella revealed: from flagellar assembly in Chlamydomonas to human obesity disorders, Cell 117 (2004), pp. 693-697.

4 E.H. Harris, The Chlamydomonas Sourcebook, Academic Press (1989).

5 P. Muller, X.P. Li and K.K. Niyogi, Non-photochemical quenching. A response to excess light energy, Plant Physiol 125 (2001), pp. 1558-1566.

6 F.A. Wollman, State transitions reveal the dynamics and flexi- 
bility of the photosynthetic apparatus, EMBO J 20 (2001), pp. 3623-3630.

7 D.R. Stevens and S. Purton, Genetic engineering of eukaryotic algae: progress and perspectives, J Phycol 33 (1997), pp. 713-722.

8 P. Kathir, M. LaVoie, W.J. Brazelton, N.A. Haas, P.A. Lefebvre and C.D. Silflow, Molecular map of the Chlamydomonas reinhardtii nuclear genome, Eukaryot Cell 2 (2003), pp. 362-379.

9•• L.A. Rymarquis, J.M. Handley, M. Thomas and D.B. Stern, Beyond complementation. Map-based cloning in Chlamydomonas reinhardtii, Plant Physiol 137 (2005), pp. 557-566.

The authors developed markers for the map-based cloning of mutant alleles. Bulked segregant analysis and marker duplexing were used to speed up the mapping process.

10 J. Rohr, N. Sarkar, S. Balenger, B.R. Jeong and H. Cerutti, Tandem inverted repeat system for selection of effective transgenic RNAi strains in Chlamydomonas, Plant J 40 (2004), pp. 611-621.

11 J.P. Davies, D.P. Weeks and A.R. Grossman, Expression of the arylsulfatase gene from the beta 2-tubulin promoter in Chlamydomonas reinhardtii, Nucleic Acids Res 20 (1992), pp. 2959-2965.

12 J. Shrager, C. Hauser, C.W. Chang, E.H. Harris, J. Davies, J. McDermott, R. Tamse, Z. Zhang and A.R. Grossman, Chlamydomonas reinhardtii genome project. A guide to the generation and use of the cDNA information, Plant Physiol 131 (2003), pp. 401-408.

13. S. Eberhard, M. Jain, C.S. Im, S. Pollock, J. Shrager, Y. Lin, A.S. Peek and A.R. Grossman, Generation of an oligonucleotide array for analysis of gene expression in Chlamydomonas reinhardtii, Curr Genet 49 (2006), pp. 106-124.

This manuscript describes the latest microarray for analysis of gene expression in C. reinhardtii, which is based on 70 mer oligonucleotides. This array represents approximately 10000 genes and the data indicate that the specificity of the array is better than that of the previous cDNA-based array.

14 E. Asamizu, K. Miura, K. Kucho, Y. Inoue, H. Fukuzawa, K. Ohyama, Y. Nakamura and S. Tabata, Generation of expressed sequence tags from low- $\mathrm{CO}_{2}$ and high- $\mathrm{CO}_{2}$ adapted cells of Chlamydomonas reinhardtii, DNA Res 7 (2000), pp. 305-307.

15 Z. Zhang, J. Shrager, M. Jain, C.W. Chang, O. Vallon and A.R. Grossman, Insights into the survival of Chlamydomonas reinhardtii during sulfur starvation based on microarray analysis of gene expression, Eukaryot Cell 3 (2004), pp. 1331-1348.

16 K. Van and M.H. Spalding, Periplasmic carbonic anhydrase structural gene (Cah1) mutant in Chlamydomonas reinhardtii, Plant Physiol 120 (1999), pp. 757-764.

17 M. Mitra, C.B. Mason, Y. Xiao, R.A. Ynalvez, S.C. Lato and J.C. Moroney, The carbonic anhydrase gene families of Chlamydomonas reinhardtii, Can J Bot 83 (2005), pp. 780-795.

18 D.T. Hanson, L.A. Franklin, G. Samuelsson and M.R. Badger, The Chlamydomonas reinhardtii cia3 mutant lacking a thylakoid lumen-localized carbonic anhydrase is limited by $\mathrm{CO}_{2}$ supply to rubisco and not photosystem II function in vivo, Plant Physiol 132 (2003), pp. 2267-2275.

19 M. Giordano, A. Norici, M. Forssen, M. Eriksson and J.A. Raven, An anaplerotic role for mitochondrial carbonic anhydrase in Chlamydomonas reinhardtii, Plant Physiol 132 (2003), pp. 2126-2134.

20 M. Mitra, S.M. Lato, R.A. Ynalvez, Y. Xiao and J.V. Moroney, Identification of a new chloroplast carbonic anhydrase in Chlamydomonas reinhardtii, Plant Physiol 135 (2004), pp. 173-182.

21 Y. Xiang, J. Zhang and D.P. Weeks, The Cia5 gene controls formation of the carbon concentrating mechanism in Chlamydomonas reinhardtii, Proc Natl Acad Sci USA 98 (2001), pp. 5341-5346.

22 H. Fukuzawa, K. Miura, K. Ishizaki, K.I. Kucho, T. Saito, T. Kohinata and $\mathrm{K}$. Ohyama, CCm1, a regulatory gene controlling the induction of a carbon-concentrating mechanism in Chlamydomonas reinhardtii by sensing $\mathrm{CO}_{2}$ availability, Proc Natl Acad Sci USA 98 (2001), pp. 5347-5352.

23 P. Vance and M.H. Spalding, Growth, photosynthesis and gene expression in Chlamydomonas over a range of $\mathrm{CO}_{2}$ concentrations and $\mathrm{CO}_{2} / \mathrm{O}_{2}$ ratios: $\mathrm{CO}_{2}$ regulates multiple acclimation states, Can J Bot 83 (2005), pp. 820-833.

24•• Y. Wang and M.H. Spalding, An inorganic carbon transport system responsible for acclimation specific to air levels of $\mathrm{CO}_{2}$ in Chlamydomonas reinhardtii, Proc Natl Acad Sci USA 103 (2006), pp. 10110-10115.

The authors identify the mutated gene responsible for the pmp1 phenotype: a marked reduction in the uptake of $\mathrm{Ci}$ when the cells are starved for $\mathrm{CO}_{2}$. The Pmp1-associated transport system is required specifically for the acclimation of cells to low $\left[\mathrm{CO}_{2}\right]$ (i.e. $0.4 \%-0.03 \% \mathrm{CO}_{2}$ ), but not to high or very low $\left[\mathrm{CO}_{2}\right]$. Furthermore, the gene affected in the mutant strain is part of a small gene family; the role of the other members of this family with respect to the CCM is being explored.

25 Z.Y. Chen, L.L. Lavigne, C.B. Mason and J.V. Moroney, Cloning and overexpression of two cDNAs encoding the low- $\mathrm{CO}_{2}-$ inducible chloroplast envelope protein LIP-36 from Chlamydomonas reinhardtii, Plant Physiol 114 (1997), pp. 265-273.

26 M.D. Burow, Z.Y. Chen, T.M. Mouton and J.V. Moroney, Isolation of cDNA clones of genes induced upon transfer of Chlamydomonas reinhardtii cells to low $\mathrm{CO}_{2}$, Plant $\mathrm{Mol}$ Biol 31 (1996), pp. 443-448.

27 S.V. Pollock, D.L. Prout, A.C. Godfrey, S.D. Lemaire and J.V. Moroney, The Chlamydomonas reinhardtii proteins Ccp1 and Ccp2 are required for long-term growth, but are not necessary for efficient photosynthesis, in a low- $\mathrm{CO}_{2}$ environment, Plant Mol Biol 56 (2004), pp. 125-132.

28 N. Rolland, A.J. Dorne, G. Amoroso, D.F. Sultemeyer, J. Joyard and J.D. Rochaix, Disruption of the plastid ycf10 open reading frame affects uptake of inorganic carbon in the chloroplast of Chlamydomonas, EMBO J 16 (1997), pp. 6713-6726.

29 J. Rexach, E. Fernandez and A. Galvan, The Chlamydomonas reinhardtii Nar1 gene encodes a chloroplast membrane protein involved in nitrite transport, Plant Cell 12 (2000), pp. 1441-1453.

30• V. Mariscal, P. Moulin, M. Orsel, A.J. Miller, E. Fernandez and A. Galvan, Differential regulation of the Chlamydomonas nar1 gene family by carbon and nitrogen, Protist 157 (2006), pp. 421-433.

This work shows that nitrite transporters appear to be regulated by $\mathrm{Ci}$ availability. The results suggest integration of nitrogen and carbon metabolism. The work also suggests that NAR1.2 might be involved in both $\mathrm{HCO}_{3}^{-}$ and $\mathrm{NO}_{2}{ }^{-}$transport.

31 C.S. Im and A.R. Grossman, Identification and regulation of high light-induced genes in Chlamydomonas reinhardtii, Plant J 30 (2002), pp. 301-313.

32 E. Soupene, N. King, E. Feild, P. Liu, K.K. Niyogi, C.H. Huang and $\mathrm{S}$. Kustu, Rhesus expression in a green alga is regulated by $\mathrm{CO}_{2}$, Proc Natl Acad Sci USA 99 (2002), pp. 7769-7773.

33 S. Kustu and W. Inwood, Biological gas channels for $\mathrm{NH}_{3}$ and $\mathrm{CO}_{2}$ : evidence that $\mathrm{Rh}$ (Rhesus) proteins are $\mathrm{CO}_{2}$ channels, Transfus Clin Biol 13 (2006), pp. 103-110.

34 M.L. Ghirardi, P.W. King, M.C. Posewitz, P.C. Maness, A. Fedorov, K. Kim, J. Cohen, K. Schulten and M. Seibert, Approaches to developing biological $\mathrm{H}_{2}$-photoproducing organisms and 
processes, Biochem Soc Trans 33 (2005), pp. 70-72.

35 O. Kruse, J. Rupprecht, K.P. Bader, S. Thomas-Hall, P.M. Schenk, G. Finazzi and B. Hankamer, Improved photobiological $\mathrm{H}_{2}$ production in engineered green algal cells, J Biol Chem 280 (2005), pp. 34170-34177.

36 A. Melis, M. Seibert and T. Happe, Genomics of green algal hydrogen research, Photosynth Res 82 (2004), pp. 277-288.

37 J.M. Quinn, M. Eriksson, J.L. Moseley and S. Merchant, Oxygen deficiency responsive gene expression in Chlamydomonas reinhardtii through a copper-sensing signal transduction pathway, Plant Physiol 128 (2002), pp. 463-471.

38 R.P. Gfeller and M. Gibbs, Fermentative metabolism of Chlamydomonas reinhardtii: I. Analysis of fermentative products from starch in dark and light, Plant Physiol 75 (1984), pp. 212-218.

39 S. Ohta, K. Miyamoto and Y. Miura, Hydrogen evolution as a consumption mode of reducing equivalents in green algal fermentation, Plant Physiol 83 (1987), pp. 1022-1026.

40•• A. Atteia, R. van Lis, G. Gelius-Dietrich, A. Adrait, J. Garin, J. Joyard, N. Rolland and W. Martin, Pyruvate formate-lyase and a novel route of eukaryotic ATP synthesis in Chlamydomonas mitochondria, J Biol Chem 281 (2006), pp. 9909-9918.

The authors completed a proteomic survey and found that Chlamydomonas makes pyruvate formate-lyase, which is almost never found in eukaryotes. Peptides of other proteins that are associated with fermentation were also identified. These findings suggest that Chlamydomonas has a range of fermentation pathways that it can use when the environment becomes anoxic.

41• A. Hemschemeier and T. Happe, The exceptional photofermentative hydrogen metabolism of the green alga Chlamydomonas reinhardtii, Biochem Soc Trans 33 (2005), pp. 39-41. Fermentative products formed by Chlamydomonas include formate, ethanol, acetate, glycerol, lactate, $\mathrm{CO}_{2}$ and $\mathrm{H}_{2}$. The authors describe interactions between fermentation, photosynthesis and $\mathrm{H}_{2}$ evolution; these interactions allow Chlamydomonas to withstand long periods of anaerobiosis. During sulfur deprivation, the cells develop photofermentative metabolism and accumulate $\mathrm{H}_{2}$.

42 M.C. Posewitz, P.W. King, S.L. Smolinski, L. Zhang, M. Seibert and M.L. Ghirardi, Discovery of two novel radical S-adenosylmethionine proteins required for the assembly of an active [Fe] hydrogenase, J Biol Chem 279 (2004), pp. 25711-25720.

43 S. Saint-Amans, L. Girbal, J. Andrade, K. Ahrens and P. Soucaille, Regulation of carbon and electron flow in Clostridium butyricum VPI 3266 grown on glucose-glycerol mixtures, J Bacteriol 183 (2001), pp. 1748-1754.

44 D.D. Wykoff, J.P. Davies, A. Melis and A.R. Grossman, The regulation of photosynthetic electron transport during nutrient deprivation in Chlamydomonas reinhardtii, Plant Physiol 117 (1998), pp. 129-139.

45 A. Melis, L. Zhang, M. Forestier, M.L. Ghirardi and M. Seibert, Sustained photobiological hydrogen gas production upon reversible inactivation of oxygen evolution in the green alga Chlamydomonas reinhardtii, Plant Physiol 122 (2000), pp. 127-136.

46 D.L. Hatfield In: D.L. Hatfield, M.J. Berry and V.N. Gladyshev, Editors, Selenium: Its Molecular Biology and Role in Human Health, Kluwer Academic Publishers (2006).
47 S.V. Novoselov, M. Rao, N.V. Onoshko, H. Zhi, G.V. Kryukov, Y. Xiang, D.P. Weeks, D.L. Hatfield and V.N. Gladyshev, Selenoproteins and selenocysteine insertion system in the model plant cell system, Chlamydomonas reinhardtii, EMBO J 21 (2002), pp. 3681-3693.

48 M. Rao, B.A. Carlson, S.V. Novoselov, D.P. Weeks, V.N. Gladyshev and D.L. Hatfield, Chlamydomonas reinhardtii selenocysteine tRNA[Ser]Sec, RNA 9 (2003), pp. 923-930.

49 H.-Y. Kim, D.E. Fomenko, Y.-E. Yoon and V.N. Gladyshev, Catalytic advantages provided by selenocysteine in methionine-S-sulfoxide reductases, Biochemistry 45 (2006), pp. 13697-13704.

50 M.T. Croft, M.J. Warren and A.G. Smith, Algae need their vitamins, Eukaryot Cell 5 (2006), pp. 1175-1183.

51• M.T. Croft, A.D. Lawrence, E. Raux-Deery, M. Warren and A.G. Smith, Algae acquire vitamin B12 through a symbiotic relationship with bacteria, Nature 483 (2005), pp. 90-93.

The authors show that vitamin B12 (cobalamin) is required by algae primarily as a cofactor for the vitamin B12-dependent methionine synthase. Furthermore, cobalamin auxotrophy arose numerous times throughout evolution, probably a consequence of the loss of the vitamin B12-independent form of the methionine synthase.

52 E. Settembre, T.P. Begley and S.E. Ealick, Structural biology of enzymes of the thiamin biosynthesis pathway, Curr Opin Struct Biol 13 (2003), pp. 739-747.

53 T. Kubodera, M. Watanabe, K. Yoshiuchi, N. Yamashita, A. Nishimura, S. Nakai, K. Gomi and H. Hanamoto, Thiamineregulated gene expression of Aspergillus oryzae thiA requires splicing of the intron containing a riboswitch-like domain in the 5'-UTR, FEBS Lett 555 (2003), pp. 516-520.

54 N. Sudarsan, J.E. Barrick and R.R. Breaker, Metabolite-binding RNA domains are present in the genes of eukaryotes, RNA 9 (2003), pp. 644-647.

55 W. Winkler, A. Nahvi and R.R. Breaker, Thiamine derivatives bind messenger RNAs directly to regulate bacterial gene expression, Nature 419 (2002), pp. 952-956.

$56 \bullet$ S.S. Merchant, M.D. Allen, J. Kropat, J.L. Moseley, J.C. Long, S. Tottey and A.M. Terauchi, Between a rock and a hard place: trace element nutrition in Chlamydomonas, Biochim Biophys Acta 1763 (2006), pp. 578-594.

Analysis of the draft genome suggests that metal homeostasis in Chlamydomonas depends upon a blend of mechanisms that operate in animals, plants and microbes. The authors discuss the ways in which Chlamydomonas acclimates to its dynamic metal environment, especially as regards to copper and iron, and provide insights into the allocation of metals in the cell, and the relationship between photo-oxidative stress and metal homeostasis.

$57 \bullet$ C. Bolling and O. Fiehn, Metabolite profiling of Chlamydomonas reinhardtii under nutrient deprivation, Plant Physiol 139 (2005), pp. 1995-2005.

The authors have developed technologies to profile metabolites in Chlamydomonas. They show distinctive metabolite profiles under nitrogen, sulfur and phosphorus deprivation conditions. This type of analysis is beginning to reveal the pathways in Chlamydomonas that are activated under specific environmental conditions. 